\title{
In Defense of a Causal Requirement on Explanation
}

\section{$\$$ Introduction}

Let us identify a causalist about scientific explanation as one who is committed, in some way or other, to the following thesis from David Lewis: "to explain an event is to provide some information about its causal history." "The tricky part, of course, is specifying what sort of causal information we are talking about. One important explication of "causal information" comes from Wesley Salmon, a causalist who developed the original and influential causalmechanical model of explanation. ${ }^{2}$ On this model, to explain an event is to provide some subset of the causal processes (and interactions between causal processes) that brought about that event. The causal-mechanical model falls under a general conception of explanation that Salmon calls the "ontic" conception-according to which explaining an event involves locating that event (the explanandum event) within certain nomologically necessitated regularities, or patterns, in the world. ${ }^{3}$ Moreover, he views these regularities as causal regularities governing interactions between causal processes. ${ }^{4}$

Salmon's approach to causal information (as information about causal processes) gives us a slightly better handle on what exactly the causalist is committed to. As a first approximation, then, in deference to Lewis and with help from Salmon, we can say that a causalist is committed to something like the following causal requirement:

The Causal Process Requirement. An explanation of an event must specify some of the causal processes that constitute that event's causal history.

${ }^{1}$ David Lewis, “Causal Explanation,” in Philosophical Papers Volume II (New York: Oxford University Press), 217.

${ }^{2}$ Salmon's view is laid out in, among other places, his "Scientific Explanation and the Causal Structure of the World," in David-Hillel Ruben, ed., Causation (New York: Oxford University Press, 1993), 78-112. For a book-length treatment of his view, see his Scientific Explanation and the Causal Structure of the World (Princeton, NJ: Princeton University Press, 1984).

${ }^{3}$ Salmon (pp. 79-81) characterizes the other conceptions of explanation as either epistemic (i.e., as arguments in which the explanandum statement follows deductively from the statements in the explanans) or modal (i.e., as exhibiting the physical necessity of the explanandum fact, given the facts in the explanans). There is reason to question this taxonomy of the different conceptions of explanation, but for now the important part is Salmon's focus on causal processes.

${ }^{4}$ For more on the important notions of 'causal process' and 'causal interaction,' see Salmon's "Causation: Production and Propagation," in Ernest Sosa and Michael Tooley, eds., Causation (New York: Oxford University Press, 1993). 
The Causal Process Requirement (CPR) is a plausible way of spelling out the general causal requirement on explanation to which all causalists are committed. ${ }^{5}$ It also provides us with an initial gloss on "providing causal information": to provide causal information about an event is to list some of the causes of that event.

If CPR is apt, then it seems reasonable to suppose that, given two competing explanations of the same event, the one that provides more causal information will be better than the other. Hence, the proponent of CPR will often be committed to this additional thesis, which I will call Proportionality:

Proportionality: Explanatory power increases in direct proportion to the amount of causal information provided. ${ }^{6}$

(As with the notion of "causal information" itself, it is difficult to specify exactly what we mean by the "amount" of causal information. I will say more about this problem below.) If providing causal information involves providing the causes themselves, then we can see that Proportionality presupposes CPR. ${ }^{7}$ (And in general we can say that the proponent of Proportionality is also committed to some variation or instantiation of the causalist requirement-whether it be CPR or some alternative formulation.) With these two theses in hand, we can define an anti-causalist as one who rejects CPR, and thus by extension Proportionality as well. Most commonly, anti-causalists base this rejection on putative counterexamples in which the best explanation of some phenomenon appears to be completely non-causal.

My claim is this: the anti-causalists are being too hasty if they let their rejection of CPR lead them to eschew causalism in general-because the causalist requirement can be

${ }^{5}$ Note that CPR is relatively weak, at least insofar as it leaves open the question of which (and how many) causal processes must be cited. Nevertheless, as we will see, there are certain explanations that do not appear to satisfy even this weak version of CPR.

${ }^{6}$ This dictum is perhaps most evident in Railton's deductive-nomological-probabilistic (DNP) account, which adverts to the "ideal explanatory text" - the full-fledged explanation of an event, including all of the relevant causal detail. For more on the DNP model and the ideal text, see Railton's "A DeductiveNomological Model of Probabilistic Explanation," Philosophy of Science 45 (1978), 206-26, reprinted in Joseph Pitt, ed., Theories of Explanation (New York: Oxford University Press, 1988).

${ }^{7}$ Although, as we will see below (cf. note 44), we can also leave open the question of whether all explanation is causal explanation and ask whether Proportionality is true if we restrict our discussion to causal explanations only. 
reformulated in a way that renders it immune to the anti-causalist challenge. Proportionality, on the other hand, turns out to be unsalvageable. The examples cited by anti-causalists do indeed show that more is not always better, when it comes to causal information; but to extend this conclusion to a complete and general rejection of causal requirements is not warranted. Instead, I argue, these examples lead us to articulate the causalist requirement in an alternative way. This alternative articulation incorporates some of the important anticausalist insights without giving up on the explanatory necessity of causal information.

As I defend my claim, I will consider what I take to be two of the strongest challenges to the causal requirement. The first challenge, which I will characterize as an "equilibrium challenge," comes from Elliott Sober. He argues that the best available explanations for the behavior of certain dynamical systems do not appear to provide any causal information, thus refuting $\mathrm{CPR} .{ }^{8}$ In response to the equilibrium challenge, I argue that, despite appearances, these equilibrium explanations are fundamentally causal. Thus, even if equilibrium explanations do not satisfy CPR, there will be an alternative formulation of the causalist thesis that does apply to those explanations. I will propose just such a formulation. I will then take the conceptual apparatus developed in response to the equilibrium challenge and apply it to instances of the second challenge, which I will characterize as an "epistemic challenge." Proponents of this challenge point out that understanding can actually be obscured when we focus on providing causal information. The insights gleaned from the equilibrium challenge provide a way of responding to the epistemic challenge as well—once again vindicating (a revised version of) the causalist thesis.

My project here is, in short, a focused attempt to trace and develop the dialectic surrounding causal requirements on scientific explanation. The general causalist requirement comes from Lewis, and is fleshed out in a particular way by Salmon. Sober presents a criticism of this fleshed out requirement, which inspires various revisions and reformulations. Along the way, I will be strengthening my defense (and revision) of the causalist requirement by pointing out how other, more recent authors have arrived at similar conclusions via different routes. I will consider, for example, James Woodward's influential manipulationist account of explanation. ${ }^{9}$ One of the lessons learned from the equilibrium

\footnotetext{
${ }^{8}$ Elliott Sober, "Equilibrium Explanations," Philosophical Studies 43 (1983), 201-10.

9 James Woodward, Making Things Happen: A Theory of Causal Explanation (New York: Oxford University Press, 2003).
} 
challenge is that an important part of the explanation of a dynamical system is a description of the system that provides some set of constraints on its behavior. These constraints will indicate what sort of factors need to be present (or absent) for the system in question to reach equilibrium. Thus, when the system satisfies these constraints, it can be characterized by a certain sort of invariance-and for Woodward, "invariance is the key to explanatoriness." ${ }^{10}$ I will also consider Michael Strevens's "kairetic" account of explanation, according to which one of the key explanatory virtues is depth. ${ }^{11}$ The equilibrium challenge forces us to reject Proportionality, and Strevens's notion of explanatory depth provides, among other insights, an elegant way of characterizing the motivation for that rejection.

\section{$\$ \quad$ Equilibrium challenges to causalism about explanation}

Perhaps the most difficult examples for causalists to deal with are those involving equilibrium explanations - which explain an observed equilibrium state of a dynamical system by providing a range of possible initial states and possible causal trajectories. Given the possible causal trajectories of the system, each of the possible initial states would have led to the observed equilibrium. ${ }^{12}$ Even if providing an explanation usually consists in providing causal information (i.e., consists in listing some of the causes of the explanandum event), equilibrium explanations appear to be an exception. They explain the equilibrium state of a dynamical system by providing a disjunction of possible causal trajectories-and, as Sober points out, "disjunctions of causal scenarios will sometimes fail to say what the cause is.",13 Thus, it would seem that we have a counterexample to CPR, in which the explanatory work is done sans causal information.

This, at least, is the argument as advanced by Sober. ${ }^{14}$ And although it may succeed against CPR as stated above, it does not succeed against an alternative formulation of the

${ }^{10}$ Ibid., 183.

${ }^{11}$ Michael Strevens, Depth: An Account of Scientific Explanation (Cambridge, MA: Harvard University Press, 2008).

${ }^{12}$ For a representation of this viewpoint regarding equilibrium explanations, see Robert W. Batterman, "Explanatory Instability," Noûs 26 (Sep 1992), 325-348.

${ }^{13}$ Sober, 205.

${ }^{14}$ Another instance of the anti-causalist strategy can be found in Ruth Berger's "Understanding Science: Why Causes Are Not Enough,” Philosophy of Science 65 (Jun 1998), 306-332. In her example, the unpredictable growth patterns of Dungeness crabs are explained by the linear distribution of their eggs. 
causalist commitment-or so I claim. In other words, the argument is too hasty if meant to apply to causal requirements in general. In order to see why, we need to have a closer look at the example Sober utilizes.

Sober's example of an equilibrium explanation involves a fitness function from population genetics. Given some population with two traits $(A$ and $B)$, the fitness function for each trait specifies the expected number of offspring for that trait, according to its frequency in the population. The system can be modeled as follows:

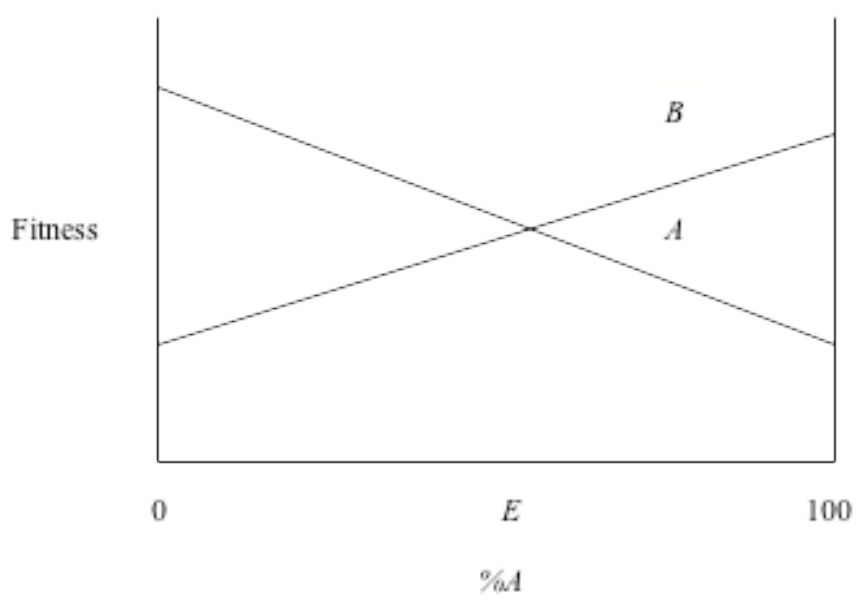

Among other things, this diagram represents the selection forces at work in this population: each of the two traits is favored by selection when it is in the minority. ${ }^{15}$ Several points on this diagram are especially salient. Note first that $E$ is an equilibrium value, at which the proportion of type $A$ to type $B$ does not change. Furthermore, $E$ is a stable equilibrium, because any deviation from $E$ will trigger selection forces that will push the system back toward E. (An unstable equilibrium would be the opposite-e.g., if the fitness functions of $A$ and $B$ were reversed. In that case, given a deviation from $E$, the selection forces would move the system away from E.) The other salient states of the system are the absorbing states, as represented by the four endpoints (or absorbing points) of the two fitness functions. If these absorbing states are reached, the forces represented in the model will not be able to

${ }^{15}$ The example, including the diagram, is taken directly from Sober, 207-8. Referring to natural selection as a "force" is somewhat contentious, but for simplicity of exposition I will nevertheless continue to do so. (But see Denis M. Walsh, Tim Lewens, and André Ariew, "The Trials of Life: Natural Selection and Random Drift," Philosophy of Science 69 (2002), 429-446.) 
move the system to a different state. In the system modeled above, then, absorbing points are points of no return.

In addition to the distinction between stable and unstable equilibria, we can also draw a distinction between global and local equilibria. This distinction is understood in terms of the range of initial conditions that will lead to the equilibrium state. ${ }^{16}$ A global equilibrium is such that the system will end up in that state no matter which initial conditions obtain, whereas a local equilibrium is such that a range of initial conditions must be specified, outside of which the system might not reach that particular equilibrium. Thus we can think of an explanation of a dynamical system in terms of the degree to which initial conditions must be specified: no specification is required in the case of global equilibria, whereas some specification is required in the case of local equilibria. And of course local equilibria themselves come in degrees - some equilibria are more local than others. So the "locality" of an equilibrium is directly proportional to the amount of information that needs to be specified, with respect to the system's initial conditions, in order to explain that system's equilibrium state. $^{17}$

We can now return to the received anti-causalist wisdom, as nicely encapsulated in the following passage from Sober.

When we are at one end of the continuum-when the equilibrium is a global one-an event can be explained in the face of considerable ignorance of the actual forces and initial conditions that in fact caused the system to be in its equilibrium state. In this circumstance, we are, in one natural sense, ignorant of the event's cause, but explanation is possible nonetheless. ${ }^{18}$

This received wisdom maintains a sharp dichotomy between causal explanation and equilibrium explanation, on the basis of which CPR, among other causal requirements, is rejected. As we will see, equilibrium explanations apparently do show that CPR is inadequate. However, rather than leading us to reject causal requirements altogether, I will attempt to show how Sober's treatment actually provides the resources for a reformulation of the causalist thesis-one which renders it immune to challenges from equilibrium explanations (and to related challenges). The crucial element of this reformulation project, as we will see below, is the claim that equilibrium explanations can actually be characterized in

\footnotetext{
${ }^{16}$ Sober, 204.

${ }^{17}$ Ibid., 208-9.

${ }^{18}$ Ibid., 209.
} 
terms of two distinct continua: in addition to the continuum of locality (i.e., the continuum of information about the initial conditions) that Sober identifies, there is also a continuum of causal information.

\section{\ Causal information}

The first step in this reformulation project is to acknowledge that, if causal information is construed in terms of the processes that constitute an event's actual causal history, then it does appear to be true that equilibrium explanations do not provide any causal information, and thus constitute a class of counterexamples to CPR. ${ }^{19}$ In the face of this problem, I propose that the causalist can modify his requirement so that it will no longer be vulnerable to such counterexamples, while at the same time retaining the spirit of causalism. I will begin fleshing out this proposal by examining the notion of the amount of causal information that an explanation provides, in light of what we have learned about equilibrium explanations. This examination will point to a replacement for CPR that is immune to the equilibrium challenge.

As noted above, it is difficult to know what exactly it means to say that one explanation provides a greater amount of causal information than another. One clear case is when the explanans of one explanation entails the explanans of a different explanation; in this case the logically stronger explanans provides a greater amount of information than the logically weaker explanans. But when there is no entailment relation, it is less clear how to compare two different explanans. ${ }^{20}$ We could begin the comparison, however, by drawing upon the resources of information theory. ${ }^{21}$ We could say that an explanans provides more information to the extent that it rules out possibilities. If we construe "causal history" as it is construed in CPR-i.e., as incorporating information about the actual causal processes

${ }^{19}$ There is room, perhaps, for the causalist to resist the conclusion that equilibrium explanations do not provide causal process information. For example, if one subscribes to something like Railton's DNP model of explanation, then one might think that a disjunction of possible causal trajectories could be incorporated into the ideal explanatory text, and thus count as causal information in some minimal sense. For a description of a similar strategy, see note 52.

${ }^{20}$ Thanks to an anonymous referee for help on this point.

${ }^{21}$ Salmon very briefly considers an information-theoretic approach to unification theories of explanation in Chapter 4 (p. 131) of his Four Decades of Scientific Explanation (Pittsburgh: University of Pittsburgh Press, 2006). Railton briefly discusses the usefulness (as well as some of the shortcomings) of information theory in his "Probability, Explanation, and Information," Synthese 48 (1981), 233-256. 
leading up to and bringing about the explanandum event-then this information-theoretic suggestion amounts to the following claim: the narrower the range of possible causal histories (of the explanandum event) allowed by the explanans, the more information it will provide. But this suggestion will not do, because explanations are always given in a context. This point is strongly emphasized in a forthcoming article by Larry Wright, and can be better understood if we consider one of his examples: an explanation of one's house burning down. ${ }^{22}$ He imagines that a contributing cause to this fire was a candle falling down on a curtain. Thus it seems straightforward to say that an explanation citing the candle falling on the curtain would provide more information than, say, an explanation merely citing the curtain's catching fire. After all, the explanation citing the candle provides more restrictions on the causal history leading up to the event (for example, it rules out the curtain's catching fire as a result of an electrical short). However, as Wright points out, the context of inquiry might affect which of the two competing explanations is more informative. For example, if the curtain has been treated with fire retardant, and we are not surprised that the candle fell on the curtain (perhaps this has happened before; perhaps it is because this has happened before that the curtain was treated with fire retardant), then even a detailed and colorful causal history of the candle's fall on the curtain might not be as informational as the simple fact that the retardant was abraded by a recent cleaning of the curtains. In short, what counts as a greater amount of causal information is going to vary from explanatory context to explanatory context. As a result, there may not be any simple, absolute, one-dimensional scale on which we can map the various "amounts" of causal information that an explanation might provide. If there were a general scale that we could use to measure differing amounts of causal information, it would likely involve several dimensions, and thus be quite complex.

Nevertheless, I think our consideration of equilibrium explanations does point us toward a helpful way of providing some restrictions on what we mean by a greater (or lesser) amount of causal information. First, however, let us try to quantify the information about initial conditions that is provided by an equilibrium explanation of a dynamical system. Recall that, because the equilibrium point $E$ in the diagram above is a stable, global

\footnotetext{
${ }^{22}$ Larry Wright, "Causal Explanations," forthcoming. The context-sensitivity of explanation is also emphasized in Michael Scriven, "Explanation, Predictions, and Laws," in Herbert Feigl and Grover Maxwell, eds., Minnesota Studies in the Philosophy of Science, Volume III: Scientific Explanation, Space, and Time (Minneapolis: University of Minnesota Press, 1962), 51-74. (See especially §3, pp. 52-3.)
} 
equilibrium, no information about the initial conditions need be provided in the equilibrium explanation (apart from the stipulation that the population cannot begin in one of the absorbing states). ${ }^{23}$ But notice that if $E$ were instead a local equilibrium, then the equilibrium explanation would have to provide a range of possible initial conditions, within which selection forces would move the system toward E. Thus, returning to information theory, we can say that in the context of the dynamical system being modeled, an explanation of a local equilibrium will provide a greater amount of information about initial conditions-will rule out more possibilities - than will an explanation of a global equilibrium. This point extends to a comparison of two local equilibria, about which we can say that the more local the equilibrium (i.e., the more detail required when specifying the initial conditions) the more information provided by the explanation. Thus, we can flesh out Sober's notion of the continuum of locality as follows. At the extreme local end of this continuum, the maximal amount of information is required: the range of initial conditions specified will consist simply of the actual initial conditions. At the other endpoint of this continuum (i.e., the point represented by global equilibria), the minimal amount of information is required: the only restriction on the initial conditions of the system is that the population cannot start in one of the absorbing states. So the equilibrium point of any given dynamical system can be mapped on this continuum according to the amount of information required when specifying the initial conditions.

Within the context of equilibrium explanations, then, we have a straightforward and illuminating way of cashing out the notion of a greater or lesser amount of information. A global equilibrium can be specified using the minimal amount of information regarding the initial conditions, whereas a maximally local equilibrium will have to be specified using the maximal amount of information: the actual initial conditions. The amount of information

\footnotetext{
${ }^{23}$ The parenthetical qualification, particularly in light of what I say below, might lead one to ask whether we have properly identified the endpoint of the continuum of information about the initial conditions. (Recall that a global equilibrium is defined as one in which there is one restriction on initial conditions, namely that the population not begin in one of the system's absorbing states.) It could be argued, for example, that there might be some dynamical systems that will reach equilibrium no matter what state they start in (which would preclude those systems from having any absorbing states). If that is correct, then the specification of a true global equilibrium need not refer to absorbing states. But such a claim would not affect my own argument. In principle I can accept either definition of 'global equilibrium,' since I am only concerned to argue that equilibrium explanations cannot appeal to an equilibrium that falls on the extreme minimal endpoint of the causal information continuum. (Thanks to an anonymous referee for helping me clarify this point.)
} 
that an equilibrium explanation provides can thus be measured in terms of the locality of the relevant equilibrium point.

So far we are in agreement with Sober. But recall that he uses this continuum to represent two kinds of information: information about initial conditions as well as information about causal forces: "When we are at one end of the continuum-when the equilibrium is a global one-an event can be explained in the face of considerable ignorance of the actual forces and initial conditions that in fact caused the system to be in its equilibrium state." ${ }^{24}$ My claim here is that we should represent these two kinds of information by positing two separate continua: one corresponding to information about initial conditions, and another corresponding to information about causal forces. If this is right, then the next natural step is an attempt to use the results of the above discussion to make more sense of the notion of a greater or lesser amount of causal information.

Here again we can learn from equilibrium explanations, which, in addition to providing information about initial conditions, also provide a disjunction of possible causal trajectories. But notice that each of the possible causal trajectories of the relevant system is a function of the initial conditions of the system and the causal forces at work in the system. In other words, equilibrium explanations refer, not just to the relevant initial conditions, but also to the relevant causal forces_-forces that, up to this point, I have been largely ignoring (in order to focus on local and global equilibria, which are distinguished according to initial conditions). But now we can (and should) extend our treatment of the initial conditions to cover the causal forces as well. For just as we can locate various equilibrium explanations on a continuum from minimal to maximal amounts of information about initial conditions (i.e., the locality continuum), we can also locate these explanations on a continuum from minimal to maximal amounts of information about the causal forces leading to equilibrium. Consider Sober's equilibrium explanation, and notice how it changes as we adjust its placement on the causal forces continuum (holding fixed its placement on the locality continuum). As we move it toward the minimal end of the causal forces continuum, we get less and less information about the causal trajectories; i.e., the explanation rules out an increasingly smaller number of the possible trajectories that might have led to the observed equilibrium. Explanations toward the maximal end of this continuum, on the other hand, will provide a

\footnotetext{
${ }^{24}$ Sober, 209. Emphasis mine.
} 
great deal of information; i.e., they will provide significant constraints on the ways in which the system reaches equilibrium. At the extreme maximal end of both continua, all possible causal trajectories will be ruled out save one: the actual causal trajectory taken through the phase space of the system in which the equilibrium was observed. Moreover, in this (admittedly unlikely) scenario, the equilibrium explanation will after all satisfy the Causal Process Requirement. Most equilibrium explanations will in fact violate CPR, but nothing about the structure of such explanations dictates that they must violate CPR. ${ }^{25}$

Let us now take stock of our progress. We have seen, first of all, that the equilibrium explanation that Sober provides does not satisfy CPR; it offers a disjunction of possible causal histories, but it does not cite any of the causal processes that constitute the actual causal history. We have also seen that in general we can describe equilibrium explanations as offering more or less information about (1) the initial conditions of the relevant dynamical system and (2) the possible combinations of causal forces leading to equilibrium. (And since the actual initial conditions and the actual causal forces together determine the actual causal trajectory of the system, we can now see that there are in fact three continua: the locality continuum, the causal forces continuum, and the causal trajectory continuum. I will largely ignore this third continuum, however, since its relevance to a given explanation is determined by that explanation's placement on the first two continua. ${ }^{26}$ ) In short, the amount of information an equilibrium explanation gives about a dynamic system is a function of where that explanation falls on both the locality continuum and the causal forces continuum. A hypothetical equilibrium explanation at the maximal information end of both continua will specify the exact causal trajectory that brought about the equilibrium-i.e., the

\footnotetext{
${ }^{25}$ Note also that since we are restricting our consideration of possible causal trajectories to a particular system, we are respecting Wright's claims about the contextual nature of explanation and avoiding the difficulties that would plague a more general information-theoretic attempt to specify the amount of causal information provided by various competing explanations.

${ }^{26}$ Thanks to an anonymous referee for help with this point. Notice also that when we recognize this dependence, we remove one barrier to identifying equilibrium explanations as causal. As noted above, even though these explanations do provide a disjunction of possible causal trajectories, it is plausible to claim that this does not count as causal information. (As Sober points out [p. 205], "causality abhors an ineliminable disjunction.") But we can grant this point and still maintain (as I do below) that equilibrium explanations provide causal information in virtue of specifying the causal forces of the relevant system. A disjunction of possible causal trajectories may not itself count as causal information, but it is derived from causal information (along with information about initial conditions). Or so I claim.
} 
actual causal processes that constituted the history of the equilibrium event. This hypothetical equilibrium explanation would satisfy CPR.

I suggest that the most reasonable move, in light of these considerations, is not a rejection of causalism but a replacement of CPR with a closely related variant of the causalist requirement. We cannot require a specification of the actual causal trajectory (i.e., we cannot require the maximum amount of information on the locality and causal forces continua), because equilibrium explanations typically explain without providing that level of detail. So why not discard CPR (and related causal requirements) entirely? Because even equilibrium explanations must provide some sort of causal information. Given that any given equilibrium explanation can be located on a continuum that represents the amount of causal information provided, the only way in which an equilibrium explanation could do its explanatory work sans causal information is if it fell on the extreme minimal endpoint of the causal forces continuum: if it provided absolutely no information about the causal forces at work in the system. Such an explanation would tell us only that there is a certain range of initial conditions needed to reach equilibrium, and when the system begins within those initial conditions, it somehow reaches equilibrium. This "explanation," however, is next to useless, as it tells us only that we are dealing with a dynamical system-a fact which presumably we already knew.

Perhaps, though, we presume too much. Perhaps an equilibrium explanation would be useful despite providing no information about the causal forces at work in the relevant system. (Imagine that the relevant system, as above, is a relatively simple one consisting of a population with two traits.) Such an explanation would identify some distribution of the population as the equilibrium state, and then provide a range of initial conditions within which the population would reach that state. From these two pieces of information (the equilibrium state and the initial conditions) would follow a third: that the population we are trying to explain is (or at least can be represented as) a dynamical system. This might seem to be enough to make the explanation a useful one. But if we consider an analogy, then I think it will become clear that the proposed (non-causal) equilibrium explanation is no good.

Consider an explanation of the state of a gas in terms of the behavior of its component molecules. While it does not seem explanatorily useful (or even feasible) to attempt to describe the causal history of any (much less all) of the component molecules, it also does not seem useful to explain the state of the gas by merely pointing out that it is 
composed of molecules, which collide with each other according to certain causal laws (e.g., the laws of Newtonian mechanics), and that those collisions somehow produce the relevant state. Intuitively, this latter explanation seems unsatisfactory. (As Woodward says, this would appear to be a "trivial, non-serious explanation of the behavior of the gas." 27 ) If this intuition is correct, then the same can be said of the equilibrium explanation that we are considering. For it seems that this trivial and non-serious explanation of the state of the gas is structurally similar to the equilibrium explanation in question. The proposed equilibrium explanation essentially says that, given certain initial conditions, features of the system somehow produce the equilibrium state. If the imagined explanation of the state of the gas is trivial and nonserious, then surely the proposed non-causal equilibrium explanation is trivial and nonserious as well.

Can we put any flesh on the bones of this triviality intuition? I think we can, if we appeal to one of Strevens's criticisms of an explanation similar to the wholly non-causal equilibrium explanation we have been considering. He claims, in short, that explanations that include black boxes are unacceptably shallow. ${ }^{28}$ (For Strevens, a black box is essentially a functional definition, "which explains $c$ 's causing $e$ by citing only the fact that $c$ has the property of being e-producing." 29 ) And it seems that the proposed non-causal equilibrium explanation is a black box explanation of the equilibrium state. It makes no claims about causes, but it does explain a system $S$ 's ending up in its equilibrium state $E$ by citing a function. This function tells us only that the system has the property of being E-producing (given certain initial conditions). We might conclude that insofar as a completely non-causal equilibrium explanation relies on a black box to do its explanatory work, it should be dismissed as shallow.

Thus, once we see that equilibrium explanations can be classified according to where they fall on a continuum representing the amount of causal information provided, we can also see that equilibrium explanations falling on the minimal endpoint of the continuum will

\footnotetext{
${ }^{27}$ James Woodward, "The Causal Mechanical Model of Explanation," in Philip Kitcher and Wesley C. Salmon, eds., Minnesota Studies in the Philosophy of Science, Volume XIII: Scientific Explanation (Minneapolis: University of Minnesota Press, 1989), 363.

${ }^{28}$ Strevens, Depth, 130-132.

${ }^{29}$ Ibid., 131.
} 
not really serve as explanations at all. Such explanations will be trivial and non-serious, not to mention shallow (among perhaps other explanatory vices).

In addition to these negative reasons for construing even equilibrium explanations as causal, there are-returning to Strevens-also positive reasons for making the same claim. Consider a different equilibrium explanation, of the fact that a ball released on the lip of a basin will end up resting at the lowest point of the basin no matter where on the lip the ball is released. Strevens points out that

while a casual inspection of the equilibrium model might give the impression that it says nothing about the particular causal process leading to the explanandum event, in fact the model is exclusively concerned to describe this very token process, but at an extremely abstract level, so abstract that the description is satisfied by every process by which the ball might have reached the bottom of the basin. ${ }^{30}$

In other words, as we move toward the minimal end of the causal information continuum, we are moving in the direction of increasingly abstract description. Nevertheless, what is being described is still a causal process (or causal force, as the case may be).

In short: we have both negative and positive reasons in support of the claim that even equilibrium explanations are in some sense causal explanations.

\section{$\$$ From causal processes to causal factors}

Since the Causal Process Requirement (CPR) is inadequate, and since we cannot entirely eschew causal information, we should reformulate the causalist requirement while insisting that an explanation provide some sort of causal information. How should we specify the sort of causal information required in a way that is general enough not to exclude equilibrium explanations? My suggestion is that we avoid reference to the actual causal history leading up to the explanandum event, and instead require simply that an explanation give us information about the causal factors that influence, in one way or another, whether or not the explanandum event occurs. (I say more about what counts as a "causal factor" below.) Thus, I propose the following alternative causalist requirement:

The Causal Factors Requirement. An explanation of an event must provide information about the causal factors that influenced whether or not that event occurred.

${ }^{30}$ Ibid., 268. 
Causal-mechanical explanations that provide actual causal history will easily meet this requirement; but what about equilibrium explanations? Well, since the function describing the behavior of the dynamical system being explained is going to represent the interplay of causal forces, it will provide some constraints, however minimal, on the ways in which the objects in the system interact with each other. Thus, it strikes me as perfectly legitimate to describe this function as providing information about the causal factors that influence whether or not the explanandum event occurs.

In fact, thinking in terms of constraints on the behavior of a system is a useful way of fleshing out the admittedly vague notion of "causal factors." Thus I also propose that we think of providing "causal information"-i.e., providing "information about causal factors"- as providing some sort of causal law governing the behavior of the system within which the explanandum event occurs. Within the context of the relevant system, an explanatory causal law, at least in the sense I intend, should indicate which combinations of causal interactions will result in the occurrence of the explanandum event, and likewise which combinations of causal interactions will fail to result in the occurrence of the event (within a certain range of initial conditions). The force of this causal law will simply be that if it is violated, then, ceteris paribus, the explanandum event will not occur. ${ }^{31}$

In Sober's equilibrium example, the fitness function, governing selection forces, is serving as the sort of causal law I am suggesting. What this function is telling us is that certain systemic changes to the selection forces (including the introduction of different forces, or the deletion of selection forces entirely) will have the result that equilibrium is not reached. When it comes to more standard causal explanations_as, for example, in Wright's explanation of the house burning down in terms of the candle falling on the curtain-the causal law will be much more specific (in keeping with that explanation's placement toward the maximal end of the causal information continuum). In this case, the information about

\footnotetext{
${ }^{31}$ The ceteris paribus clause is designed to address, among other things, the concern that this counterfactual construal of causal laws is too strong - because there could be backup processes, operating according to different laws, that would bring about the explanandum event even if the explanatorily relevant causal factors were subtracted. In the context of Sober's equilibrium explanation, for example, mutation could serve as a backup process: a mutation could occur which would move the population toward equilibrium even if the selection forces were altered significantly. Thus, one of the "other conditions remaining the same" is the absence of mutation (cf. p. 207, where Sober explicitly rules out mutation as part of the model of the system). Thanks to an anonymous referee for bringing this concern to my attention.
} 
causal factors is telling us that, holding certain initial conditions fixed, the house will not burn (ceteris paribus) unless the candle falls on the curtain.

My suggestion here resembles something that Woodward says in his important and influential book, Making Things Happen. ${ }^{32}$ He rejects any sort of nomothetic model of explanation according to which explanation involves subsumption under laws, but he does want to appeal to explanatory generalizations-where a generalization counts as explanatory if it is invariant in the right way. (For Woodward, recall, invariance is the key to explanatoriness.) Although Woodward's treatment of invariance is certainly worth considering in more detail, all I will say here is that for him a generalization has the right kind of invariance if it represents a pattern of counterfactual dependence-which is to say that it is stable under some specifiable range of interventions. ${ }^{33}$ The "causal laws" that, I claim, are required for explanation share this invariance that Woodward finds crucial to explanatoriness, but they also include a contextual parameter that indicates which features of the relevant system must be held fixed (e.g., the initial conditions) in order for the necessary invariance to obtain.

The schema I am proposing, then, is one in which an explanation will provide more or less information about the initial conditions of the relevant system or context, and more or less-but some-information about the causal forces at work in that system. At this point it is worth noting that the causal requirement I favor suggests a model of explanation that closely resembles the one Hempel and Oppenheim proposed in their groundbreaking work on scientific explanation. ${ }^{34}$ Recall, briefly, that their deductive-nomological (DN) model of explanation required a set of explanans statements, which included a statement of the antecedent conditions and a statement corresponding to each applicable general law. An explanation consisted in providing these explanans statements such that the explananduma sentence describing the phenomenon to be explained-could be logically derived from the explanans. Although I do not wish to attempt a rehabilitation of the DN model (the

\footnotetext{
32 James Woodward, Making Things Happen. Woodward's theory is a causal theory, but he is not interested in arguing that all explanation is causal in nature; instead, he explicitly restricts his discussion to causal explanations. Nevertheless (p. 6), he thinks that Sober's explanation would count as causal by his criteria.

${ }^{33}$ Ibid., 17. See also Chapter 6 for Woodward's extended discussion of invariance.

${ }^{34}$ Carl G. Hempel and Paul Oppenheim, "Studies in the Logic of Explanation," in Joseph Pitt, ed., Theories of Explanation (New York: Oxford University Press, 1988), 9-50.
} 
counterexamples are numerous and well-established), I do find it interesting that consideration of equilibrium explanations, as providing a challenge to models of explanation that endorse CPR, leads us to reformulate the causalist requirement in a way that evokes the DN model. The important difference, of course, is that my proposal replaces the DN model's inadequate notion of a "general law" with a notion that requires causal information (and more closely resembles a generalization with the right sort of invariance [in Woodward's sense]). But whereas a view that endorses CPR requires that the causal information come in the form of information about the actual causal history of the explanandum event, my proposal (CFR) does not require this. Instead, my proposal allows the required causal information to be represented in a wide variety of ways, depending upon the nature of the explanandum. The causal information might (and often will) come in the form of a causal history, in which case the causal law will be very specific. But the relevant causal law might also prescind from individual causal processes in order to make a more abstract statement about the system in question: it might come in the form of a fitness function, or a probabilistic description of molecular movement, or some other specification that represents a move toward the minimal end of the causal information continuum. (This flexibility of representation is in part what I am trying to suggest by using the "causal factors" locution.) To summarize, we might say that in moving from CPR to CFR, we have moved from providing the causes themselves to providing information about those causes. Along the way, we have learned a few lessons from Sober's treatment of equilibrium explanations. The first lesson is one we should have learned from the DN model: initial conditions are important. The second lesson that we learn from equilibrium explanations is that CPR is inadequate. Together, these two lessons suggest CFR-which replaces CPR while preserving the importance of both initial conditions and causal information.

The revised causal requirement I am suggesting also has affinities with certain aspects of Strevens's kairetic account of explanation. One of the building blocks of his account is a "causal model," which has the form of a DN explanation but also "purports to represent a chain of causal influence running from the states of affairs identified by the premises to the event identified by the conclusion." ${ }^{35}$ Thus, Strevens's approach, like mine, is crucially different from the DN approach in that the relevant entailment represents a causal,

\footnotetext{
${ }^{35}$ Strevens, Depth, 72. (His discussion of the notion of a causal model begins on p. 71.)
} 
rather than a logical relation. ${ }^{36}$ But we take different routes to this destination. Strevens begins with some of the famous counterexamples to the DN model, and points out that, in these cases, there is an explanatory asymmetry where there is no logical asymmetry. ${ }^{37}$ The basis for this asymmetry is the causal relation, which suggests causal entailment rather than logical entailment. I, on the other hand, have built in causal relations from the beginning, and attempted to show that these causal relations are an essential part of even equilibrium explanations. In so doing, I have revised the causalist requirement in a way that evokes the DN model.

We are now in a position to see whether the revised causal requirement CFR can hold up in the face of further challenges. But before we move beyond the equilibrium challenge, I would like to draw out a third lesson from equilibrium explanations.

\section{$\mathbb{S E x p l a n a t o r y}$ depth and the rejection of the Proportionality thesis}

The third thing we learn from equilibrium explanations is that we should jettison the Proportionality thesis. In order to see why, let us return once again to the dual continua that characterize an equilibrium explanation: information about initial conditions and information about causal forces. In both cases, less is more; in both cases, reducing the amount of information increases the power of the explanation. If the equilibrium is global, then minimal specification of initial conditions is necessary; in this case, we have an explanation that applies (almost) no matter what the system's initial state is. ${ }^{38}$ To provide more information about the initial conditions is to offer an explanation that does not apply as broadly, and hence is not as powerful. And the same holds for the causal information continuum: at least some causal information is required, but the more general the causal law, the more possible trajectories (and hence states of the system) will be covered by the explanation. A more specific causal law, which provides more information about (and hence

\footnotetext{
${ }^{36}$ Ibid., 92-93.

${ }^{37}$ Strevens discusses this in ibid., §1.4. The two examples he considers are the famous flagpole
} and barometer examples. From the length of a flagpole's shadow, together with the position of the sun (along with some laws about how light behaves), we can logically deduce the height of the flagpole; but the length of its shadow (etc.) does not explain the height of the flagpole. Similarly, we can deduce from a certain barometer reading that a storm is approaching; but the barometer reading does not explain the occurrence of the storm. In both of these cases, what is wrong with the logical derivation is that it runs counter to the direction of causation.

${ }^{38}$ See note 23 for a discussion of the parenthetical qualification. 
more restrictions on) the possible causal pathways leading to the explanandum event, will not apply as broadly and thus will not be as powerful.

There are various ways to understand this claim that, contra the Proportionality thesis, a more general causal law is preferable. The first is in terms of the unification approach to explanation. According to unification approaches, an explanation is better insofar as it has greater unifying power; and one of the key elements of unifying power is generality. ${ }^{39}$ Despite the fruitfulness of thinking about explanation as unification, I will focus on a different way of understanding the claim: in terms of explanatory depth.

Another way to put the point, then, is to say that the virtue of explanatory depth sometimes conflicts with the Proportionality requirement. And in cases of conflict, we should choose greater explanatory depth over greater amounts of causal information. And just what is explanatory depth? Strevens provides an elegant characterization:

Explanations having depth ... strip away vast quantities of apparently relevant, large-scale causal detail, showing thereby that the phenomenon to be explained depends on only a kind of "deep causal structure" of the system in question, a structure that is deep now not because it is so physical (though it is that) but because it is so abstract. The salient but irrelevant causal details are the shallows, then, and the more abstract—-that is, more general—properties of the system are its depths, fleshed out by the details but inconsequentially so. ${ }^{40}$

As Strevens points out, equilibrium explanations in particular represent the ideal of explanatory depth,

combining as they do two monumental abstractions: first, the abstraction of a high-level dynamics from the physical underpinnings, ... and second, in the equilibrium stage, the abstraction of a certain even higher-level property of the dynamics — the universality of a particular end point-from the high-level dynamics obtained in the first step. ${ }^{41}$

Thus, consideration of doubly and elegantly abstract equilibrium explanations, in light of the explanatory virtue of depth, leads us to reject Proportionality.

${ }^{39}$ For more on unification approaches to explanation, see Michael Friedman, "Explanation and Scientific Understanding," Journal of Philosophy 71 (1974), 5-19. See also two articles from Philip Kitcher: "Explanatory Unification," Philosophy of Science 48 (1981), 507-531; and "Explanatory Unification and the Causal Structure of the World," in P. Kitcher and W. C. Salmon, eds., Minnesota Studies in the Philosophy of Science, Volume XIII: Scientific Explanation (Minneapolis: University of Minnesota Press, 1989), 410-505.

${ }^{40}$ Strevens, Depth, 137. Strevens also advocates (pp. 147-148) small tradeoffs in the accuracy of a particular explanatory model in exchange for greater generality-i.e., greater explanatory depth.

${ }^{41}$ Ibid., 137. 
Of course, one need not go quite so far in rejecting Proportionality. One could pursue a more ecumenical or pluralistic approach to the question of whether higher-level explanations are preferable to lower-level explanations (which provide more causal information at the expense of explanatory depth). Frank Jackson and Philip Pettit, for example, argue that the choice between the two levels of explanation is pragmatic: whether one prefers the higher- or lower-level explanation depends on one's perspective or purpose. "Explanations of different levels provide complementary bodies of information on one and the same topic; we do not throw any explanation away just because we have access to another." ${ }^{43}$ Sober himself also advocates a similar sort of pluralism, when he points out that

higher-level sciences "abstract away" from the physical details that make for differences among the micro-realizations that a given higher-level property possesses. However, this does not make higherlevel explanations "better" in any absolute sense. ... The reductionist claim that lower-level explanations are always better and the antireductionist claim that they are always worse are both mistaken. ${ }^{44}$

I join these authors in rejecting Proportionality. I also lean toward Strevens's view, according to which depth (or generality, or a higher level of abstraction) is a greater explanatory virtue than causal detail-but that tendency is not essential to my defense of causalism. The causalist can opt for a pluralist approach instead.

\section{$\$ \quad$ Applying the Causal Factors Requirement}

If what I have said in response to the challenge from equilibrium explanations is right, then we should expect a parallel response (i.e., a response that appeals to CFR) to succeed in the

${ }^{42}$ Frank Jackson and Philip Pettit, "In Defense of Explanatory Ecumenism," Economics and Philosophy 8 (1992), 1-21. They endorse causalism about explanation (cf. p. 13), but they argue against "explanatory or methodological fundamentalism" (p. 7), which always recommends the lower-level explanation and thus considers a micro-physical explanation to be objectively superior. Insofar as lowerlevel explanations provide greater amounts of causal detail, explanatory fundamentalists will be very much in sympathy with proponents of Proportionality.

${ }^{43}$ Jackson and Pettit, 16.

${ }^{44}$ Elliott Sober, "The Multiple Realizability Argument against Reductionism," Philosophy of Science 66 (1999), 542-564. Insofar as I have construed Proportionality as presupposing CPR (or some alternate formulation of the causalist requirement), then it is obvious that Sober rejects Proportionalitysince he rejects CPR on the basis of equilibrium explanations. But in this article he is restricting his discussion to causal explanations, and even in that context he rejects (or would reject) Proportionality. 
face of other sorts of challenges to CPR. Thus, as a way of vetting my proposal, I will briefly consider two examples of another type of challenge.

The first example I will consider involves another dynamical system, but the challenge is more of an epistemic challenge, rather than an equilibrium challenge. The basic point of this challenge is that additional causal detail can actually obscure understanding, and hence an explanation ${ }^{45}$ - especially when one wants to use the explanation in question as a part of practical reasoning. Alan Garfinkel uses an example from population ecology to make the point clear:

Suppose we have an ecological system composed of foxes and rabbits. There are periodic fluctuations in the population levels of the two species, and the explanation turns out to be that the foxes eat the rabbits to such a point that there are too few rabbits left to sustain the fox population, so the foxes begin dying off. After a while, this takes the pressure off the rabbits, who then begin to multiply until there is plenty of food for the foxes, who begin to multiply, killing more rabbits, and so forth. ${ }^{46}$

As Garfinkel points out, a rabbit that is trying to avoid a predator should not be too interested in an explanation of another rabbit's death that includes the specific details of the causal history of that rabbit's death (eaten by which fox, at what time, etc.). In fact, the particular fox and the particular time do not make a difference; had it not been that fox, chances are good (given a large enough fox population) that it would have been another. ${ }^{47} \mathrm{~A}$ more useful explanation would instead point to the large fox population as a whole. Additional causal detail is irrelevant and therefore unhelpful.

Garfinkel's example is clearly telling against Proportionality. An explanation of a rabbit's death that cites the large fox population provides less causal information than an explanation that cites the specific causal trajectory leading up to the event. And yet this

\footnotetext{
${ }^{45} \mathrm{I}$ am not here advocating an identification between explanation and understanding, merely pointing out that one way to weaken an explanation is to reduce the amount of understanding it generates.

${ }^{46}$ Alan Garfinkel, Forms of Explanation: Rethinking the Questions in Social Theory (New Haven, CT: Yale University Press, 1991), 53. Garfinkel is arguing specifically against reductionism (pp. 53-57), but his reasons for eschewing reductionism also militate against Proportionality. Hilary Putnam also argues against reductionism, particularly in his "Philosophy and our Mental Life," in Mind, Language, and Reality (Cambridge: Cambridge University Press, 1975), 291-303. Putnam says (p. 296) that a micro-story about why a square peg won't fit in a round hole is either a terrible explanation or no explanation at all.
}

${ }^{47}$ In certain "difference-making” respects, Garfinkel's approach anticipates Strevens's kairetic account of explanation, especially as laid out in his "The Causal and Unification Approaches to Explanation Unified - Causally," Noûs 38:1 (2004), 154-76 (material from which was incorporated into Depth). 
explanation, given that it applies to a much broader range of rabbit deaths (and would be more useful for rabbits trying to avoid getting eaten [or humans trying to preserve the rabbit population]), is at the same time more powerful than an alternative explanation that provides a greater amount of causal information. It is, in short, the deeper of the two alternatives. Moreover, as also evidenced in this example, less causal information can be better if it allows us to explain not only what happened, but what could have happened, had certain things been otherwise. The higher-order explanation of the rabbit's death (in terms of the large fox population) makes it plain that had the first fox not eaten him, the second (or third, etc.) likely would have. Similarly, as we saw above, Sober's equilibrium explanation is superior to a maximal causal detail (i.e., actual causal history) explanation: it explains not just why the population took the actual trajectory it did, but why the population would have still ended up at the same place, even if it had taken a different trajectory. If Proportionality were correct, then the explanation at the maximal causal detail end would be the better explanation; but, as we have seen, it is clearly not. Sometimes, then, pace proponents of Proportionality, less causal detail makes for a better causal explanation-in particular, when less causal detail allows us to pick out modal features of the system being explained.

It seems, then, that we have another good reason to give up on Proportionality. Are these considerations from Garfinkel similarly telling against CPR? And what about CFR? Hopefully it is clear by now that even if the answer to the first question is "Yes," the answer to the second is "No." As we have already noted, the best explanation of the rabbit's death (i.e., the one that is most useful for those concerned with keeping rabbits safe from predators) is one that abstracts away from causal history, and instead points to a structural feature of the system: the large fox population. Hence, the best explanation of this system violates CPR, and thus endangers causalism - unless there is an alternative to CPR that can accommodate examples such as Garfinkel's. Fortunately for the causalists, there is such an alternative, and it is represented by CFR - which, recall, says that explanation requires only some information about the causal factors influencing the occurrence of the explanandum event (about the "causal laws" governing the behavior of the system). And this is precisely what Garfinkel's explanation is providing. His explanation is telling us that without a certain distribution of rabbit and fox populations, the rabbit's death would not have occurred (or, more precisely, it says that a certain number of rabbit deaths would not have occurred.) The behaviors being modeled-predation and reproduction chief among them-most certainly 
involve causal processes, which means that information about the various relationships between those behaviors reasonably counts as information about the relevant causal factors. It seems, then, that this explanation of the rabbit's death satisfies CFR for much the same reason that Sober's equilibrium explanation satisfies CFR.

Another example of an epistemic challenge comes from an earlier article by Woodward, who is addressing Salmon's causal-mechanical model of explanation (as utilized above when formulating $\mathrm{CPR}){ }^{48}$ His criticism thus pertains to both CPR and Proportionality:

More also needs to be said about how Salmon's model applies to complex physical systems which involve large numbers of interactions among many distinct fundamental causal processes. In such cases it is often hopeless to try to understand the behavior of the whole system by tracing each individual process. Instead one needs to find a way of representing what the system does on the whole or on average, which abstracts from such specific causal detail.49

Woodward goes on to apply this point to an explanation of the behavior of a gas that appeals to the ideal gas laws. The reason why the ideal gas laws are useful for explaining the behavior of a gas is precisely because they omit (abstract from) the individual causal processes that constitute the gas's behavior. With respect to a particular state of the gas, we could say that the ideal gas laws abstract from the individual causal processes that constitute the causal history of that state.

Notice first of all that Woodward makes another strong case against Proportionality. The explanation of the behavior of a gas is yet another example of many in which (1) there are two competing explanations, (2) which differ markedly in terms of the amount of causal information they provide, and yet (3) the explanation with less causal information is clearly the superior explanation (i.e., the explanation with greater power). In other words, there will be many cases in which, again, it would be "hopeless to try to understand the behavior of the whole system by tracing each individual process." ${ }^{50}$ And since tracing individual processes is precisely what CPR requires, it seems that Woodward's example is also telling against CPR.

As with Garfinkel's case, however, a retreat to CFR remains available. If we consider the behavior of the gas in question as the behavior of a system, then an explanation of any

\footnotetext{
${ }^{48}$ See note 2.

${ }^{49}$ Woodward, "The Causal Mechanical Model of Explanation," 362-363.

${ }^{50}$ Ibid.
} 
particular state must posit some restriction on the initial conditions, and moreover will posit the ideal gas laws as accurately governing the behavior of this system. Considered in light of the continuum of causal information, it does appear that this explanation provides less causal information than does, for example, Sober's equilibrium explanation. Nonetheless, the interactions represented by the gas laws are causal interactions, and thus even this explanation is not completely devoid of causal information. CFR once again appears vindicated.

There might be a concern here that the move I am suggesting is just as trivial and non-serious as the move we saw Woodward criticizing above. ${ }^{51}$ According to Woodward, recall, a putative explanation of the behavior of a gas that posits molecules that collide with each other according to the laws of Newtonian mechanics-and says only that these collisions somehow produce the behavior in question-is a trivial and non-serious causalist explanation. (We also saw that an equilibrium explanation completely devoid of causal information is akin to this trivial and non-serious explanation of the behavior of a gas.) Since Woodward directs this criticism against a (hypothetical) causalist view that appeals to an objectionable form of abstraction, it might seem as though my own proposal is vulnerable to this criticism as well. But it is not. To see why, first recall the equilibrium explanations considered above. The causal approach I am suggesting does not replace those equilibrium explanations with corresponding causal explanations; rather, it simply points out that equilibrium explanations (or at least the ones in the examples given) are already causal to begin with. So the point I am making here is not analogous to any attempt to provide an abstract (but unhelpful) version of a "causal processes" explanation. Instead, I am suggesting a slightly, but crucially, different treatment of Woodward's example. This treatment points out that the best explanation of the behavior of a gas, whatever that turns out to be, is already causal to begin with. This is because the equation(s) used in the best explanation, much like the fitness function in Sober's equilibrium explanation, represent causal processes. The causal information that satisfies CFR can be abstracted away from, or selectively highlighted, or what have you; but such information remains, even if under the surface, a crucial part of any explanation. The causal nature of explanation is inescapable.

${ }^{51}$ Ibid., 363. 


\section{$\$$ Conclusion}

I will conclude by briefly returning to Sober's equilibrium explanation. It is true that his example, and related examples, do cause trouble for certain formulations of the causal requirement on explanation - in particular, the Causal Process Requirement. However, as I have tried to show, Sober's distinction between local and global equilibria provides an explanatory framework that we can fruitfully extend by taking into account the amount of causal detail as regards not just initial conditions, but also causal forces. And if we do take into account causal forces, then once we are told that the graph is about selection, there is a sense in which we have already got a causal explanation. Moreover, this point complements certain themes in Woodward and Strevens-two different authors who have converged upon a similar conclusion via different routes.

Therefore Sober's equilibrium explanation, contrary to the received wisdom, is after all a causal explanation (in virtue of its reference to selection forces). Whether he originally intended it or not, Sober appears to have pointed the way toward a causal gloss on equilibrium explanation. ${ }^{52}$ Moreover, this treatment of equilibrium explanations can be extended to other sorts of explanations in a way that supports a revised causal requirement-the Causal Factors Requirement. ${ }^{53}$

${ }^{52}$ Sober has acknowledged as much in personal correspondence. Zachary Ernst, in his defense of Railton's DNP model ("Evolutionary Game Theory and the Origins of Fairness," PhD. dissertation, 2002), provides another causal gloss on this type of equilibrium explanation: he construes it as providing information about the "ideal explanatory text" (which is a crucial element of the DNP model; see note 6), and thus causal even if indirectly so.

${ }^{53}$ I would like to thank Zachary Ernst, Eric Schwitzgebel, and two anonymous referees for Oxford University Press for helpful comments on earlier drafts. I would also like to thank André Ariew (who nurtured this paper in its infancy) and Erich Reck (who stepped in and helped out during the difficult teenage years) for many detailed and helpful comments on numerous drafts - as well as extended discussion that has greatly increased my understanding of these issues. 